\title{
Fixing of photorefractive volume holograms in $\mathrm{K}_{1-y} \mathrm{Li}_{\mathbf{y}} \mathrm{Ta}_{1-x} \mathrm{O}_{3}$
}

\author{
Victor Leyva \\ Arroyo Optics, Santa Monica, California 90404 \\ Doruk Engin, Xiaolin Tong, Min Tong, and Amnon Yariv \\ California Institute of Technology, Pasadena, California 91125 \\ Aharon Agranat \\ Hebrew University of Jerusalem, Jerusalem, Israel
}

\begin{abstract}
Received December 23, 1994
We report the fixing of photorefractive holographic gratings with high efficiency in a sample of $\mathrm{K}_{1-y} \mathrm{Li}_{y} \mathrm{Ta}_{1-x} \mathrm{Nb}_{x} \mathrm{O}_{3}$ doped with $\mathrm{Cu}, \mathrm{V}$, and $\mathrm{Ti}$. Holograms are thermally fixed through the screening of a photorefractive space-charge field by a nonphotoactive species at elevated temperatures. Fixed holograms are revealed by illumination at lower temperatures. Diffraction efficiencies of $25 \%$ in a 0.54 -cm-thick sample are measured. Holograms undergo thermal decay with a $0.67-\mathrm{eV}$ activation energy.
\end{abstract}

The process by which a photorefractive holographic grating is converted to one that is not erased under illumination is referred to as fixing. The ability to fix gratings with high efficiencies and long lifetimes is essential for holographic archival data storage applications and for the fabrication of thick holographic optical elements. Thermal fixing refers to the process by which a photorefractive space-charge grating is compensated at elevated temperatures by a mobile nonphotoactive species. At lower temperatures the thermally fixed sample is illuminated, partially erasing the initial photorefractive grating and revealing a fixed grating unaffected by illumination. Thermal fixing has been observed in ilmenite materials $\left(\mathrm{LiNbO}_{3}\right.$, $\mathrm{LiTaO}_{3}, \mathrm{Ba}_{2} \mathrm{NaNb}_{5} \mathrm{O}_{15}$ ) and in the perovskite material $\mathrm{KNbO}_{3} .{ }^{1,2}$ High fixed efficiencies of $98 \%$ have been achieved in a 1-cm-thick $\mathrm{LiNbO}_{3}$ sample. ${ }^{3}$ However, $\mathrm{LiNbO}_{3}$ exhibits low photorefractive sensitivity, ${ }^{4}$ making it unsuitable for many holographic data storage applications. Large values of photorefractive sensitivities have been reported in $\mathrm{KNbO}_{3}$ (Ref. 4); however, reported fixing efficiencies are less than $1 \%$ in a 0.2 -cm-thick sample. ${ }^{2}$ Materials are needed with both high photorefractive sensitivity and high fixing efficiency.

$\mathrm{K}_{1-y} \mathrm{Li}_{y} \mathrm{Ta}_{1-x} \mathrm{Nb}_{x} \mathrm{O}_{3}$ (KLTN) is a ferroelectric perovskite material. ${ }^{5}$ The addition of $\mathrm{Li}$ to $\mathrm{KTa}_{1-x} \mathrm{Nb}_{x} \mathrm{O}_{3}$ (KTN) has been found to improve the optical quality of crystals with phase transitions near room temperature. The ferroelectric phase transition occurs at a temperature that increases with both $\mathrm{Li}$ and $\mathrm{Nb}$ concentrations. Large quadratic electro-optic effects occur in the cubic paraelectric region above the phase transition, which results in high-diffraction-efficiency holograms. In this region of operation, an external electric field is required for the generation of a firstorder grating that can diffract the writing beams. The field allows for full and rapid amplitude modulation of the diffraction efficiency of the previously recorded holograms. ${ }^{6}$

A KLTN crystal doped with $\mathrm{Cu}, \mathrm{V}$, and $\mathrm{Ti}$ was grown by the top-seeded solution growth method. The crystal is grown with an excess of $\mathrm{K}$ in the flux. The ratio of $\mathrm{Li}$ to $\mathrm{K}$ in the flux is 0.027 , and the ratio of $\mathrm{Ta}$ to $\mathrm{Nb}$ in the flux is 0.43. A sample measuring $5.4 \mathrm{~mm} \times 5.7 \mathrm{~mm} \times 8.0 \mathrm{~mm}$ was cut and polished along the crystallographic axes. The $\mathrm{OH}^{-}$vibrational absorption band at $3512 \mathrm{~cm}^{-1}$ was measured with a Fourier-transform infrared spectrometer to have a peak magnitude of $0.41 \mathrm{~cm}^{-1}$ and an integrated intensity of $57.6 \mathrm{~cm}^{-2}$. With the calibration results of Scherban et al., ${ }^{7}$ this corresponds to a proton concentration of $5.76 \times 10^{-5}$ mole fraction or $9.2 \times 10^{17} \mathrm{~cm}^{-3}$. Metal electrodes were evaporated on the $5.4 \mathrm{~mm} \times 5.7 \mathrm{~mm}$ faces. The sample was placed on a thermoelectric temperature-controlled mount enclosed in a vacuum chamber. The temperature dependence of the dielectric constant was determined from measurements of the low-frequency capacitance of the sample. A peak occurred at $T=-17^{\circ} \mathrm{C}$, which corresponds to the ferroelectric phase transition temperature. All holographic measurements were done above this temperature in the cubic paraelectric phase.

We wrote holographic gratings using two beams with $175-\mathrm{mW} / \mathrm{cm}^{2}$ intensity from an Ar laser operating at $488 \mathrm{~nm}$. The beams were expanded to completely illuminate the sample and were ordinary polarized in order to minimize coupling effects, including beam fanning. The full angle between the writing beams was $28 \mathrm{deg}$, which resulted in a grating period of $1.0 \mu \mathrm{m}$. An external field was applied in the plane of incidence. The diffraction efficiency of the grating was monitored with a weak extraordinarily polarized beam from a $\mathrm{He}-\mathrm{Ne}$ laser aligned at the Bragg angle. The diffraction efficiency is defined as the ratio of the diffracted to incident intensities corrected for surface 


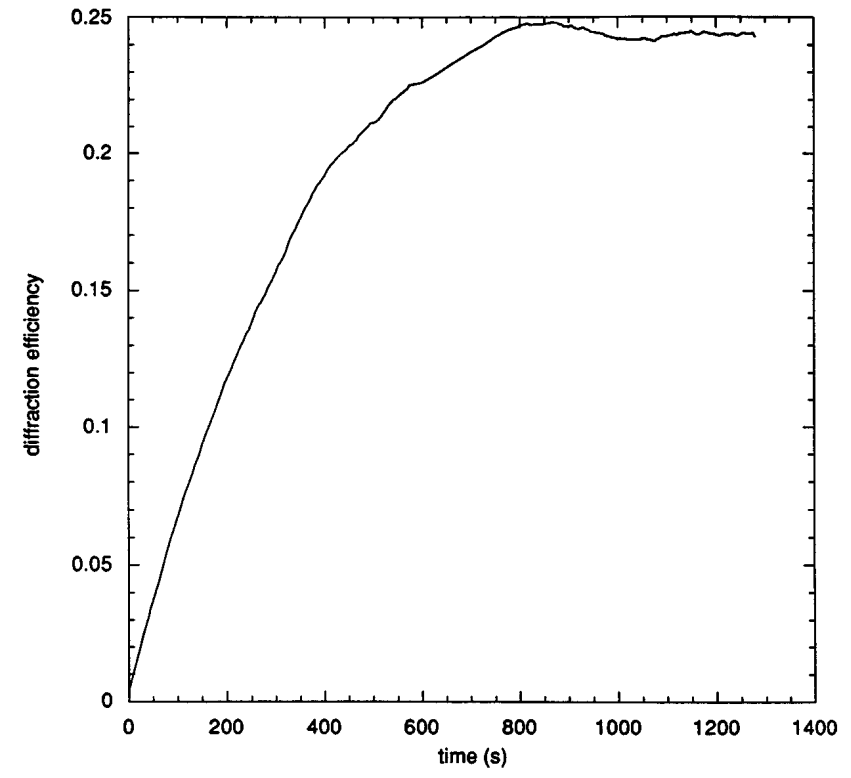

Fig. 1. Revealing of a fixed hologram in KLTN at $T=5{ }^{\circ} \mathrm{C}$ under illumination with a $488-\mathrm{nm}$ beam of $175-\mathrm{mW} / \mathrm{cm}^{2}$ intensity. The fixed diffraction efficiency reached a value of $25 \%$.

reflections. To prevent optical erasure of the grating, we attenuated the $\mathrm{He}-\mathrm{Ne}$ beam to $10^{-6} \mathrm{~W}$, and a computer-controlled shutter was used to block the beam between measurements.

Holograms could be fixed by either a sequential or a simultaneous process. In the sequential method holograms are written at or below room temperature. The sample is then heated in the dark to an elevated temperature, typically $70^{\circ} \mathrm{C}$ for $10 \mathrm{~min}$, during which the space-charge field is screened. At a lower temperature the fixed hologram is revealed under illumination. The strongest holograms were produced by the simultaneous process in which the screening of the hologram occurs during the writing stage at an elevated temperature. ${ }^{1,8}$ Writing at elevated temperatures generates larger space-charge modulations because of the lack of a buildup of a net space-charge field, which would oppose the charge transport required for grating formation. Results for this process are described as follows. The sample was heated to $70{ }^{\circ} \mathrm{C}$, and a field of $2000 \mathrm{~V} / \mathrm{cm}$ was applied. The sample was then illuminated with the 488-nm writing beams for a period of $15 \mathrm{~min}$. After writing, the sample was cooled in the dark to $5^{\circ} \mathrm{C}$. A small residual diffraction efficiency of less than $1 \%$ was measured. The fixed grating was revealed by illuminating the sample with a $488-\mathrm{nm} 175-\mathrm{mW} / \mathrm{cm}^{2}$ beam. The beam was ordinarily polarized and angularly offset from the writing angle to prevent self-enhancement effects. The fixed diffraction efficiency increased to a value of $25 \%$ over a period of $10 \mathrm{~min}$ (Fig. 1). The hologram is fixed and decays at a rate independent of illumination. The fixing and development process is similar to that observed in $\mathrm{LiNbO}_{3}{ }^{1}$

The overall efficiency of the fixing process was investigated. A hologram with a diffraction efficiency of $17 \%$ was stored at $5{ }^{\circ} \mathrm{C}$ with an applied field of $2000 \mathrm{~V} / \mathrm{cm}$. The sample was then heated to $70^{\circ} \mathrm{C}$ for
$10 \mathrm{~min}$ and then returned to $5^{\circ} \mathrm{C}$. The fixed hologram was revealed under illumination with an applied field of $2000 \mathrm{~V} / \mathrm{cm}$. The fixed diffraction efficiency reached a value of $11 \%$. The refractive-index change of the hologram for extraordinarily polarized light was $\Delta n=1.75 \times 10^{-5}$ before fixing and $\Delta n=1.38 \times 10^{-5}$ after fixing. Almost $80 \%$ conversion of the electronic grating to a fixed grating is achieved. This indicates that electronic dark conduction, which would lead to thermal erasure of the optically written grating at the fixing temperature, can be neglected. Unlike in $\mathrm{LiNbO}_{3}$ and $\mathrm{KNbO}_{3}$, there is no photovoltaic effect in cubic KLTN. The diffusion forces on photoexcited charge carriers and the externally applied field act to reveal the fixed grating effectively.

The fixed holograms were observed to decay at a rate that is independent of illumination. Neglecting the electronic dark conductivity, the decay time for fixing with an ionic species is essentially given by the dielectric relaxation time of the sample ${ }^{9,10}$ :

$$
\tau=\left(\omega_{i}+D_{i} K^{2}+i K \mu_{i} E_{0}\right)^{-1},
$$

where $\omega=\left(e \mu_{i} n_{i 0} / \varepsilon\right)$ is the ionic dielectric relaxation frequency, $\mu_{i}$ is the ionic mobility, $n_{i 0}$ is the ionic density, $\varepsilon$ is the dielectric constant, $D_{i}$ is the ionic diffusion constant, $K$ is the grating wave vector, and $E_{0}$ is the applied field. Hologram decay measurements were made in the temperature range of -10 to $30^{\circ} \mathrm{C}$. At each temperature a hologram was fixed and its decay monitored with the weak $\mathrm{He}-\mathrm{Ne}$ beam from which the exponential decay time of the refractiveindex grating was determined. Results are presented in Fig. 2 and obey an Arrhenius dependence of the form

$$
\tau(T)=t_{0} \exp \left(E_{A} / k_{B} T\right) \text {. }
$$

An activation energy of $E_{A}=0.67 \pm 0.03 \mathrm{eV}$ and a constant of $t_{0}=(6.64+1.12) \times 10^{-9} \mathrm{~s}$ were fitted to the decay measurements. At $T=20^{\circ} \mathrm{C}$ the calculated decay time is $36 \mathrm{~min}$. Holograms could not be redeveloped after erasure. This suggests that the decay

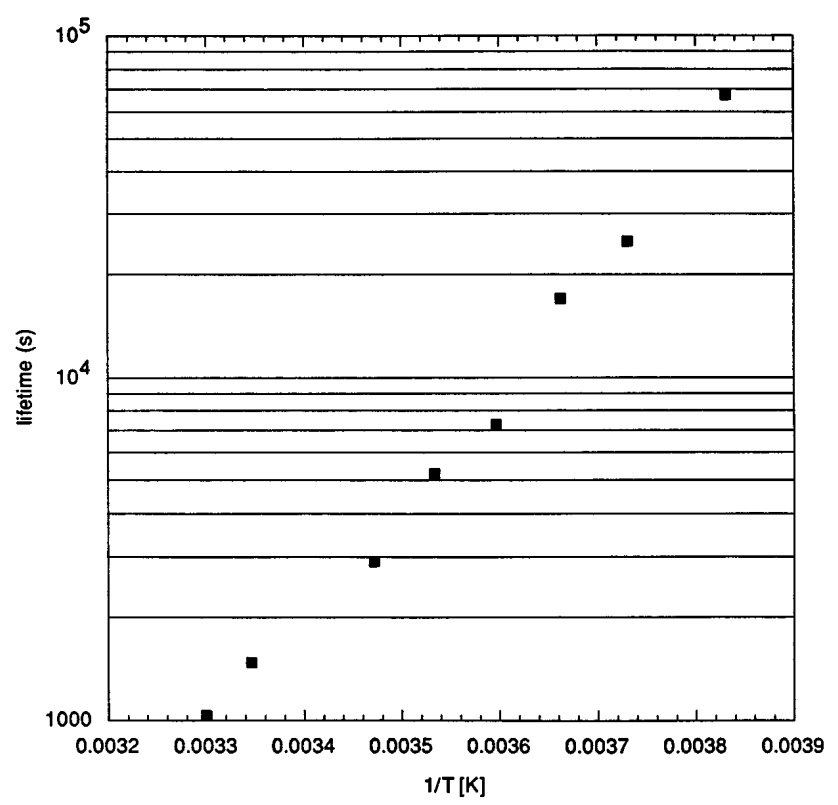

Fig. 2. Thermal decay lifetimes of fixed holograms in KLTN. The lifetimes display an Arrhenius dependence with an activation an energy of $E_{A}=0.67 \pm 0.03 \mathrm{eV}$. 
mechanism is due to a relaxation of the nonphotoactive (ionic) grating and not to the reformation of a compensating electronic grating. In this temperature region the conduction of the fixing species dominates the electronic dark conduction. As at elevated temperatures, a photorefractive grating kept in the dark at lower temperatures will fix at rate given in Fig. 2.

Ionic conduction necessary for thermal fixing has been observed in a number of materials. Protonic conduction, in which protons migrate by hopping from an $\mathrm{O}^{2-}$ ion to an adjacent one, occurs in $\mathrm{LiNbO}_{3}$ and $\mathrm{KTaO}_{3}$. Protons were identified as the mobile species responsible for fixing in $\mathrm{LiNbO}_{3} .{ }^{11,12}$ The activation energy for the conductivity is $E_{A}=1.23 \mathrm{eV}$. Scherban et al. investigated the conductivity in samples of $\mathrm{KTaO}_{3}{ }^{7}$ In samples that were not oxidized, including one with an $\mathrm{OH}^{-}$concentration nearly identical to that of the KLTN sample, protonic conduction dominated at elevated temperatures, with an activation energy of $0.73 \mathrm{eV}$. A dependence of activation energies on dopant concentration was observed in Fe-doped samples and was attributed to the effects of protons associating with dopant ions. The fixing process in $\mathrm{KNbO}_{3}$ exhibits an activation energy of $1.0 \mathrm{eV}$, though the fixing species was not identified. ${ }^{2}$ KLTN is expected to exhibit conduction processes similar to those observed in $\mathrm{KTaO}_{3}$ and $\mathrm{KNbO}_{3}$. The nearly identical proton concentrations and activation energies for KLTN and $\mathrm{KTaO}_{3}$ suggest that protons may be the fixing species in our sample. In addition to protonic conduction, KLTN is expected to show an increased ionic conduction of unknown origin in the region near the phase transition, as observed in KTN. ${ }^{13}$ If protons are the fixing species, fixed lifetimes can be increased by decreasing the concentration of protons. There is a limit to how far this approach can be taken. A certain minimum concentration is required for screening the photorefractive space-charge field during the fixing process. The concentration $\left[\mathrm{OH}^{-}\right]=9.2 \times 10^{17} \mathrm{~cm}^{-3}$ determined for the KLTN sample may allow for an order-of-magnitude increase in lifetime.

In summary, we have demonstrated fixing of holograms with high efficiency in a sample of KLTN. Ther- mal fixing occurs by screening of a photorefractive space-charge field by a mobile species at elevated temperatures followed by revealing of the fixed grating under illumination at lower temperatures. A diffraction efficiency of $25 \%$ in a 0.54 -cm-thick sample was measured. Near $80 \%$ conversion of the optically written electronic grating to a fixed grating is achieved. The thermal decay of the hologram exhibited an Arrhenius type dependence with an $E_{A}=0.67 \pm 0.03 \mathrm{eV}$ activation energy.

The authors acknowledge the support of the Advanced Research Projects Agency University Research Initiative under U.S. Office of Naval Research grant N00014-92-J-1891.

\section{References}

1. J. Amodei and D. Staebler, Appl. Phys. Lett. 18, 540 (1971).

2. G. Montemezzani and P. Günter, J. Opt. Soc. Am. B 7, 2323 (1990).

3. V. Leyva, G. A. Rakuljic, and B. O'Conner, Appl Phys. Lett. 65, 1079 (1994).

4. P. Günter, Phys. Rep. 93, 199 (1982).

5. A. Agranat, R. Hofmeister, and A. Yariv, Opt. Lett. 17, 713 (1992).

6. A. Agranat, V. Leyva, and A. Yariv, Opt. Lett. 14, 1017 (1989).

7. T. Scherban, A. S. Nowick, L. A. Boatner, and M. M. Abraham, Appl. Phys. A 55, 324 (1992).

8. G. Montemezzani, M. Zgonik, and P. Günter, J. Opt. Soc. Am. B 10, 171 (1993).

9. A. Yariv, V. Leyva, and G. A. Rakuljic, in Digest of Conference on Nonlinear Optics: Materials, Fundamentals, and Applications (Optical Society of America, Washington, D.C., 1994).

10. A. Yariv, S. Orlov, G. A. Rakuljic, and V. Leyva, Opt. Lett. 20, 1334 (1995).

11. H. Vormann, G. Weber, S. Kapphan, and E. Kratzig, Solid State Commun. 40, 543 (1981).

12. S. Klauer, M. Wohlecke, and S. Kapphan, Phys. Rev. B 45, 2786 (1992).

13. I. Krishan, K. K. Srivastava, and K. Lal, J. Phys. C 8, 71 (1975). 